\title{
Design of a Realistic PET-CT-MRI Phantom
}

\author{
Vincent Keereman, Pieter Mollet, Yves Fierens, Samuel Espana, Stefaan Vandenberghe
}

\begin{abstract}
The validation of the PET image quality of new PET-MRI systems should be done against the image quality of currently available PET-CT systems. This includes the validation of new attenuation correction methods. Such validation studies should preferentially be done using a phantom. There are currently no phantoms that have a realistic appearance on PET, CT and MRI. In this work we present the design and evaluation of such a phantom.

The four most important tissue types for attenuation correction are air, lung, soft tissue and bone. An attenuation correction phantom should therefore contain these four tissue types. As it is difficult to mimic bone and lung on all three modalities using a synthetic material, we propose the use of biological material obtained from cadavers. For the lung section a lobe of a pig lung was used. It was excised and inflated using a ventilator. For the bone section the middle section of a bovine femur was used. Both parts were fixed inside a PMMA cylinder with radius $10 \mathrm{~cm}$. The phantom was filled with ${ }^{18}$ F-FDG and two hot spheres and one cold sphere were added.
\end{abstract}

First a PET scan was acquired on a PET-CT system. Subsequently, a transmission measurement and a $\mathrm{CT}$ acquisition were done on the same system. Afterwards, the phantom was moved to the MRI facility and a UTE-MRI was acquired. Average CT values and MRI $R_{2}$ values in bone and lung were calculated to evaluate the realistic appearance of the phantom on both modalities. The PET data was reconstructed with CT-based, transmission-based and MRI-based attenuation correction. The activity in the hot and cold spheres in the images reconstructed using transmission-based and MRI-based attenuation correction was compared to the reconstructed activity using CT-based attenuation correction.

The average CT values in lung and bone were $-630 \mathrm{HU}$ and $1300 \mathrm{HU}$ respectively. The average $R_{2}$ values were $0.7 \mathrm{~ms}^{-1}$ and $1.05 \mathrm{~ms}^{-1}$ respectively. These values are comparable to the values observed in clinical data sets. Transmission-based and MRI-based attenuation correction yielded an average difference with CTbased attenuation correction in the hot spots of $-22 \%$ and $-8 \%$. In the cold spot the average differences were $+3 \%$ and $-8 \%$.

The construction of a PET-CT-MRI phantom was described. The phantom has a realistic appearance on all three modalities. It was used to evaluate two attenuation correction methods for PET-MRI scanners. tion.

Index Terms-PET, CT, MRI, PET-MRI, attenuation correc-

\section{INTRODUCTION}

$\mathbf{I}$ $\mathrm{N}$ the last few years there have been many developments in the field of PET-MRI. The first brain PET insert for a clinical MRI system was installed by Siemens in 2006[1]. Philips has developed a sequential PET-MRI system, the first version of which was installed in 2009[2]. At the end of 2010, the first fully integrated whole-body PET-MRI system

V. Keereman, P. Mollet, S. Espana and S. Vandenberghe are with Department of Electronics and Information Systems, MEDISIP, Ghent UniversityIBBT-IBiTech, De Pintelaan 185 Block B, 9000 Gent, Belgium (email: Vincent.Keereman@ugent.be)

Y. Fierens is with Radiology/BEFY, UZ Brussel, Laarbeeklaan 101, 1090 Brussel, Belgium

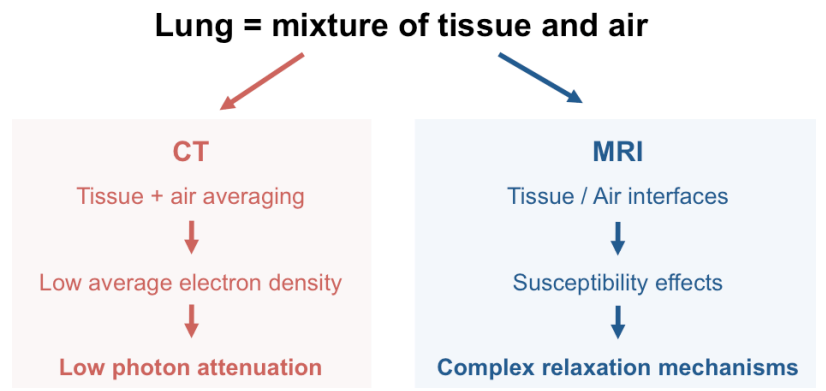

Figure 1. Diagram illustrating the origin of the specific properties of lung on CT and MRI images.

was presented by Siemens. Extensive research is currently being done to develop new PET-MRI designs, e.g. in the HYPERimage and SUBLIMA consortia[3].

While PET-MRI will enable a number of new applications, the validation of the PET image quality of new PET-MRI systems should be done against the image quality of currently available PET systems. This includes the validation of the different corrections implemented in PET reconstruction, such as attenuation correction. Attenuation correction in PET-CT or standalone PET systems is done based on the CT image or on an image acquired with a rotating transmission source (mostly ${ }^{137} \mathrm{Cs}$ ). Both methods will most likely not be used for derivation of the attenuation map in new PET-MRI systems. The attenuation map could then be derived from MRI images. This is difficult as MRI signals are not directly related to photon attenuation but are governed by the proton density and relaxation properties of tissue. On conventional MRI images, there is also no signal in bone and lung, making it difficult to distinguish these tissue types from air. Different methods have been proposed to derive the attenuation map from MRI images, such as template matching, pattern recognition or the use of specialized MRI (UTE) sequences[4], [5], [6]. A method for deriving the attenuation map from simultaneous transmission and emission imaging using an annulus source has also been proposed recently[7].

As these methods are new, their accuracy should be validated against the attenuation correction used in PET-CT or standalone PET. This could be done using phantom or human data. There are some disadvantages to using human data. First of all, it is often difficult to obtain these data, in part due to the radiation dose delivered to human volunteers during PET and/or CT acquisitions. Second, reproducibility between different scanners is limited, as movement of the volunteer between different scanners may lead to inaccurate coregistration. As no two patients are the same, it is also impossible to compare acquisitions of different subjects. Another problem is caused by the presence of physiological motion of the heart 


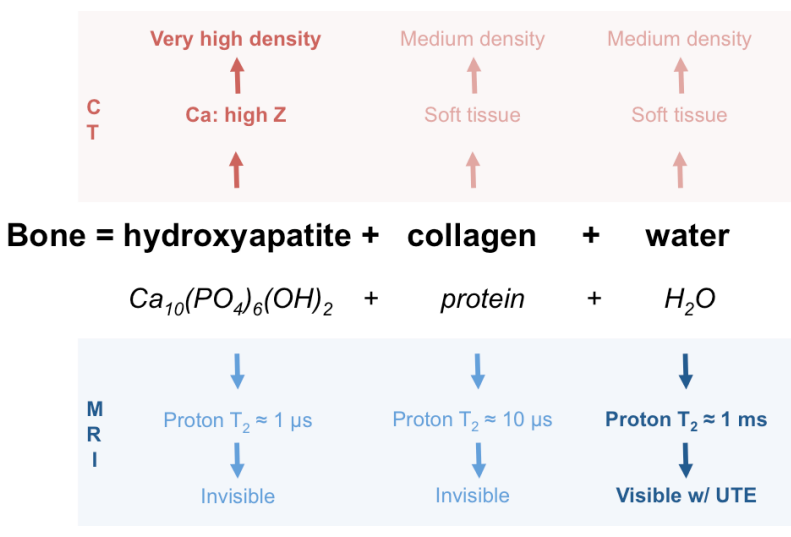

Figure 2. Diagram illustrating the origin of the specific properties of cortical bone on CT and MRI images.

and lungs, as the effect of motion can not easily be separated from the effect of attenuation.

Therefore, it would be better to use a phantom for the validation of new attenuation correction methods for PETMRI. The four most important tissue types for attenuation correction are air, lung, soft tissue and bone[8]. A suitable phantom needs to mimic these four tissue types to allow evaluation of attenuation correction methods. This means that the materials used in the phantom should have a realistic physical response to PET, CT and MRI. However, there are currently no phantoms available that fulfill these requirements. The goal of this work is to develop such a phantom. Before describing the construction of the phantom, the problems that arise in the design of such a phantom are first explained.

Although air and soft tissue can be emulated by air and water respectively on all three modalities, mimicking lung and bone is much more difficult. This is due to the fact that the specific properties of these tissue types on image modalities using ionizing radiation (PET, CT) are determined by other properties of the tissue than the properties that determine their appearance on MRI. More specifically, the relevant tissue property for CT (and PET) is electron density. For MRI, the appearance is determined by the proton density and relaxation properties of the tissue. The relaxation properties are defined by the longitudinal $\left(T_{1}\right)$ and transverse $\left(T_{2}\right)$ relaxation times.

First, we describe the origin of the CT and MRI image properties of lung tissue. This is illustrated in fig. 1. The lungs consist of millions of alveoli, which can be seen as small spheres of tissue filled with air. The low density of lung on CT is due to the averaging of the photon attenuation through air and through tissue. On the other hand, the complex relaxation mechanisms that occur in lung tissue in MRI imaging are due to susceptibility effects, caused by the difference in susceptibility between tissue and air. At each tissue interface, small differences in magnetic field are created by these susceptibility differences. These lead to faster relaxation of the protons close to a tissue interfaces.

The origin of the CT and MRI properties of bone is illustrated in fig. 2. Bone consists of 3 parts: hydroxyapatite, collagen and water. Hydroxyapatite $\left(\mathrm{Ca}_{10}\left(\mathrm{PO}_{4}\right)_{6}(\mathrm{OH})_{2}\right)$, also
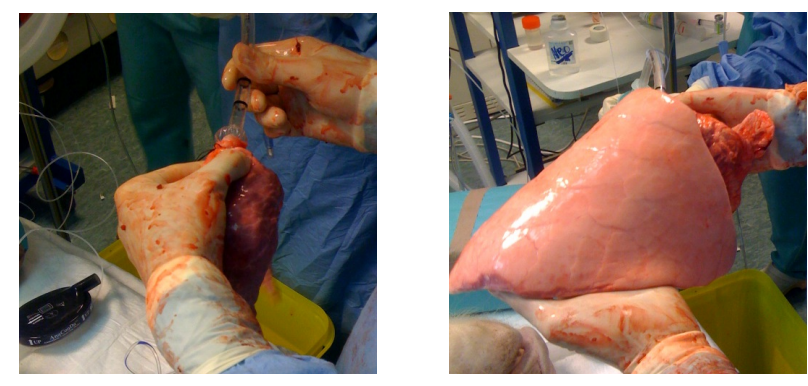

Figure 3. The collapsed (left) and reinflated lung (right).

called bone mineral, causes the high density of bone on CT because of the calcium atoms it contains. The specific properties of bone on MRI, mainly a very short $T_{2}$ relaxation time $(\approx 1 \mathrm{~ms})$, are caused by the protons in water. The protons in hydroxyapatite and collagen have $T_{2}$ relaxation times of less than $10 \mu \mathrm{s}$ and are invisible for MRI.

This shows that the specific properties of bone and lung on CT (or PET) and MRI images are due to entirely different mechanisms. Therefore, it is difficult to find synthetic materials which mimic bone and lung on all three modalities. In the next sections of this work we will first describe the construction of the phantom. Then, the appearance of the phantom on CT and MRI will be evaluated to assess if it is realistic. Finally, we will use the phantom to compare three attenuation correction methods.

\section{MATERIALS AND METHODS}

\section{A. Phantom construction}

We propose to use biological material obtained from cadavers for the bone and lung parts of the phantom. This avoids the difficulty of finding synthetic materials which accurately represent lung and bone. A lobe of a pig lung was obtained from a pig sacrificed after a cardiac surgery experiment. As a lung is maintained open inside the body by the negative pressure within the chest cavity, it collapses once it is exposed to normal air pressure. Therefore it had to be reinflated mechanically. A cannula was inserted into the main bronchus of the lobe and fixed air-tight. The lung was inflated using a positive pressure ventilator available in the lab (fig. 3). Once the lung was inflated the cannula was sealed air-tight and the lung was submersed in $4 \%$ formaldehyde solution for fixation. This allows long-term storage of biological tissue. Formaldehyde also leads to altering of the protein structure of the lung, reducing the elasticity of the lung tissue.

For the bone part, a bovine femur bone was obtained from a local slaughterhouse. A piece from the middle of the shaft was cut out. The bone has a thick cortical layer $(\approx 10 \mathrm{~mm})$ and a center filled with bone marrow. The marrow was removed from the cylinder as it is not relevant for our research. This yielded a piece of cortical bone approximately cylindrically shaped with inner radius $\approx 10 \mathrm{~mm}$, outer radius $\approx 20 \mathrm{~mm}$ and height $\approx 80 \mathrm{~mm}$. It was also stored in $4 \%$ formaldehyde solution.

A PMMA cylinder of a Jacszak phantom was used as a base for the phantom. 4 CT-MRI markers were attached to 


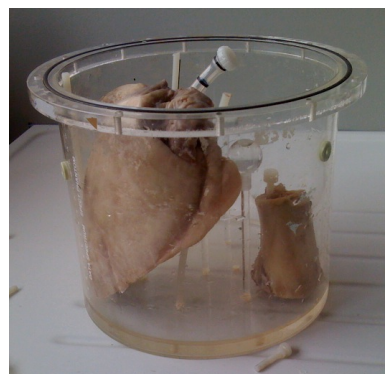

Figure 4. The assembled phantom before it was filled with water.

the surface of the cylinder to allow coregistration between CT and MRI. The bone was screwed to the floor of the cylinder with a plastic screw. The lung was fixed to three plastic rods inserted in the floor of the cylinder using suture wire. Two hot spheres with $10 \mathrm{~mm}$ radius were filled with ${ }^{18} \mathrm{~F}-\mathrm{FDG}$ and inserted. A cold sphere was also inserted. The phantom was then filled with water with ${ }^{18} \mathrm{~F}$-FDG. The ratio of the activity concentration in the hot spheres to the background was 8:1. The total activity inside the phantom was approximately 2 $\mathrm{mCi}$. Fig. 4 shows a picture of the assembled phantom.

\section{B. Acquisitions}

4 different acquisitions were done. First a standard low-dose CT (50 mAs, $120 \mathrm{kVp}$ ) was acquired on the Philips Gemini TF system (Philips Medical Systems, Cleveland, USA). Then a PET was acquired on the same system. Then a simultaneous emission and transmission PET acquisition, using an annulus source as described in [7], was performed. The acquisition time for both PET acquisitions was 5 minutes. The last scan was a UTE MRI scan on a Siemens Magnetom 3T system (Siemens, Erlangen, Germany). The UTE sequence acquires two images with echo times $0.08 \mathrm{~ms}$ and $3.2 \mathrm{~ms}$ respectively. The first image, which is not a real echo but an image reconstructed from the free inductive decay (FID) signal, is acquired very quickly after RF excitation and allows visualization of lung and bone. The second image, which is a normal gradient echo image, is acquired at a time point when the signal in lung and bone has almost entirely disappeared.

\section{Derivation of attenuation maps}

The CT image was reconstructed using the software provided by the manufacturer. A CT-based attenuation map for $511 \mathrm{keV}$ photons was derived using bilinear scaling as described in [9]:

$$
\begin{aligned}
\mu\left(\mathrm{cm}^{-1}\right) & =0.097 \frac{H U+1000}{1000} & & H U \leq 0 \\
& =0.097+0.057 \frac{H U}{1000} & & H U>0
\end{aligned}
$$

The transmission-based attenuation map was derived from the simultaneous emission and transmission measurement using the method described in [7]. Detailed explanation of this method is outside the scope of this work, but it is based on the principle that the coincidences from the annulus source and from the patient can be separated using Time-Of-Flight (TOF) information. The attenuation map can then be reconstructed from the data obtained from the annulus source.

Both MRI images were coregistered to the CT image using the alignment wizard implemented in AMIDE[10]. The four CT-MRI markers were used as fiducial markers. As the PET and CT scans were acquired on the same bed and the phantom was not moved, this yields MRI images that are coregistered to the PET images as well. A MRI-based attenuation map was derived using a method comparable to a previous publication from our group[6]. It is based on segmentation of the transverse relaxation rate map $\left(R_{2}\right.$-map), which can be calculated from both images acquired with the UTE sequence on a voxelby-voxel basis:

$$
R_{2}=\frac{\ln \left(I_{1}\right)-\ln \left(I_{2}\right)}{T_{E 2}-T_{E 1}}
$$

where $I_{1}$ and $I_{2}$ are the MRI image intensities in the FID and gradient echo images respectively and $T_{E 1}$ and $T_{E 2}$ are the echo times of both images. The method described in [6] only discriminates bone, soft tissue and air. The method used in this work was modified to also discriminate lung based on the $R_{2}$ of lung, which is slightly lower than the $R_{2}$ of cortical bone. The attenuation map was derived from the $R_{2}$-map using a simple mapping:

$$
\begin{aligned}
& \mu\left(\mathrm{cm}^{-1}\right)=0 \quad R_{2}=0 \quad \text { (air) } \\
& =0.097 \\
& =0.03 \\
& 0<R_{2} \leq 0.5 \\
& 0.5<R_{2} \leq 0.9 \\
& R_{2}>0.9 \\
& \text { (soft tissue) } \\
& \text { (lung) } \\
& \text { (bone) }
\end{aligned}
$$

\section{PET reconstruction}

Reconstruction of the PET images was done using a 3D listmode ML-EM reconstruction algorithm with attenuation correction[11]. Three different attenuation maps were used: CT-based, transmission-based and MRI-based. The images were reconstructed to a $122 \times 122 \times 32$ matrix with $4 \mathrm{~mm}$ isotropic voxel size. The reconstruction process was stopped after 50 iterations.

\section{E. Evaluation}

A spherical volume of interest (VOI) with $10 \mathrm{~mm}$ radius was drawn in the lung and in the bone. The average values inside these VOIs on the CT and the $R_{2}$-map were calculated to verify if the materials in the phantom have realistic values. 3 VOIs with $10 \mathrm{~mm}$ radius were drawn over the spheres in the reconstructed PET images. The total activities in the spheres with transmission-based and MR-based attenuation correction were compared to the total activity in the spheres with CTbased attenuation correction.

\section{RESULTS}

\section{A. CT and MRI properties}

Fig. 5 shows the same slice of the first and second echo of the MRI image and the $R_{2}$-map. Fig. 6 shows a sagittal slice of the CT image, the attenuation map derived from the transmission scan and the MRI-based attenuation map. The 


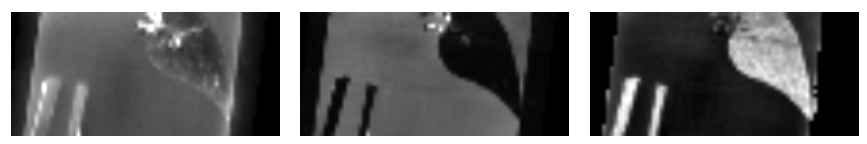

Figure 5. Sagittal slice of the first (left) and second (middle) echo MRI image and the $R_{2}$-map derived from them (right).

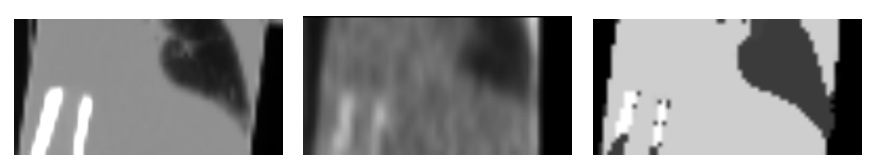

Figure 6. Sagittal slice of the CT (left), transmission-based attenuation map (middle) and MRI-based attenuation map.

average value of the lung and bone on the $\mathrm{CT}$ was $-630 \mathrm{HU}$ and $1300 \mathrm{HU}$ respectively. The average $R_{2}$ value of lung and bone was $0.7 \mathrm{~ms}^{-1}$ and $1.05 \mathrm{~ms}^{-1}$ respectively.

\section{B. Reconstructed PET images}

Transverse slices of the PET images reconstructed with the different attenuation maps are shown in fig. 7. Compared to CT-based attenuation correction, there was an average underestimation of the activity in the hot spots of $22 \%$ using the transmission-based attenuation correction and $8 \%$ using the MRI-based attenuation correction respectively. In the cold spot there was an overestimation of $3 \%$ and an underestimation of $8 \%$ respectively.

\section{Discussion}

The CT depicted in fig. 6 shows low density in the lung, medium density in the water and high density in the bone. The average $\mathrm{HU}$ values in the bone and lung are also comparable to what is found in clinical data sets. Therefore, we can conclude that the materials used in this phantom realistically represent lung, soft tissue and bone on CT. We can expect from these results that the $511 \mathrm{keV}$ photons of PET will also interact realistically with these materials.

In the FID image, shown on the left of fig. 5, signal is detected in the bone and lung. On the gradient echo image, which is shown in the middle of fig. 5, the signal in lung and bone has almost completely disappeared. This is also observed when a UTE-MRI image is acquired of a human thorax. The $R_{2}$ values that are calculated are also comparable to what can be found in clinical images. This means that the appearance of the bone and lung on MRI is realistic.
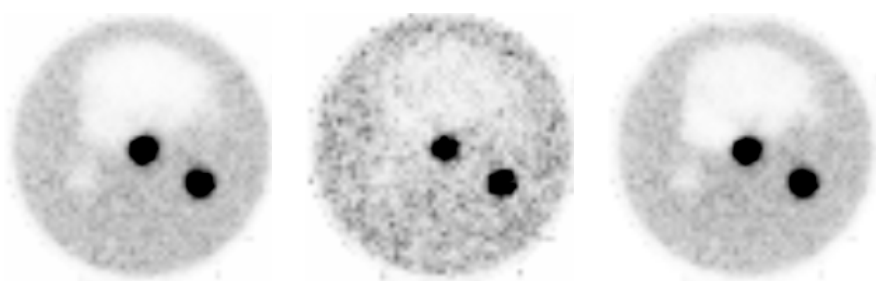

Figure 7. Transverse slice of PET images reconstructed with CT-based (left), transmission-based (middle) and MRI-based (right) attenuation correction
Therefore, we can conclude from the CT and UTE-MRI images that the materials used in this phantom provide a good substitute and that this phantom can be used to evaluate new attenuation correction methods for PET-MRI.

The performance of two different attenuation correction methods usable for PET-MRI was evaluated using the phantom. Segmented MRI-based attenuation correction performs better than the transmission-based attenuation correction. There also seems to be an underestimation of the attenuation coefficient of soft tissue in the transmission-based attenuation map. This is probably because no corrections for scatter or count rate effects were implemented in the reconstruction of the transmission-based attenuation map. As the evaluation of these attenuation correction methods is merely intended here as an example of an application of this phantom, further discussion on the cause for these errors is outside the scope of this work.

The evaluation of attenuation correction methods, as described here, is a possible application of this phantom. A normal antropomorphic PET-CT phantom could not be used as the lung and bone section would not provide a realistic MRI signal. A normal MRI phantom could not be used as there are no MRI phantoms that contain a lung or bone section. However, there are some limitations to this phantom. First of all, methods that rely on the anatomical structure of the human body to determine the attenuation map will fail on this phantom, as it does not mimic typical human anatomical constitution. This means that template-based or atlas-based methods can not be evaluated. Another limitation of this phantom is that it can not be exactly reproduced. If a new comparable phantom is created, the lung and bone will always slightly differ in shape from the lung and bone used in this phantom. A third problem is that neither the lung nor the bone can be filled with activity. This is not entirely realistic, as there will also be tracer uptake in the lung and bone for most tracers. In that case, the effect of errors in the attenuation map situated in the lung or bone will be larger. Nevertheless, this phantom currently provides the only option to evaluate new MRI-based attenuation correction methods on non-human data.

Other possible applications of this phantom can be conceived. It could be used to evaluate other corrections implemented on PET scanners, such as scatter correction. Evaluation of the correct registration of MRI and PET is also possible, although a simpler phantom might be more useful for this. It could also be used for the performance evaluation of UTE MRI sequences. Another possibility would be to adjust this phantom to allow breathing motion of the lungs. It could then be used to evaluate MRI-based motion correction methods for PET or MRI, which are currently being investigated. This would require a new fixation method, as fixation with formaldehyde changes the elastic properties of lung tissue so that it becomes much less elastic and difficult to inflate or deflate.

\section{CONClusion}

The construction of a PET-CT-MRI phantom was described. The phantom has a realistic appearance on all three modalities 
and can be used to evaluate the performance of new attenuation correction methods for PET-MRI. As an example, a MRIbased and transmission-based attenuation correction method were compared to CT-based attenuation correction. The MRIbased attenuation correction method currently performs better, mainly due to the fact that no scatter or count rate corrections were implemented in the transmission based method.

\section{ACKNOWLEDGEMENTS}

This work was supported by the EU FP7 projects HYPERimage (Grant Agreement $\mathrm{Nr}$ 201651) and SUBLIMA (Grant Agreement $\mathrm{Nr}$ 241711). The authors want to thank Dr. Thierry Bové, Dr. Stefaan Bouchez and Mieke

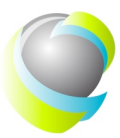

SUBLIMA Olieslagers for their help.

\section{REFERENCES}

[1] H Schlemmer, B Pichler, M Schmand, Z Burbar, C Michel, R Ladebeck, K Jattke, D Townsend, C Nahmias, P Jacob, W Heiss, and C Claussen, "Simultaneous mr/pet imaging of the human brain: Feasibility study," Radiology, vol. 248, no. 3, pp. 1028-35, Jan 2008.

[2] N Ojha, J Griesmer, Zhiqiang Hu, L Shao, D Izquierdo, J Machac, O Ratib, H Zaidi, V Fuster, and Z Fayad, "Pet performance of the gemini tf pet - mr: The world's first whole body pet - mri scanner," IEEE Nucl Sci Conf R, pp. 2013 - 2015, 2010.

[3] V Schulz, T Solf, B Weissler, P Gebhardt, P Fischer, M Ritzert, V Mlotok, C Piemonte, N Zorzi, M Melchiorri, S Vandenberghe, $\mathrm{V}$ Keereman, T Schaeffter, and P Marsden, "A preclinical pet/mr insert for a human 3t mr scanner," IEEE Nucl Sci Conf R, pp. 2577 - 2579, 2009.

[4] M Hofmann, F Steinke, and V Scheel, "Mri-based attenuation correction for pet/mri: a novel approach combining pattern recognition and atlas registration," J Nucl Med, vol. 49, pp. 1875-83, Jan 2008.

[5] E Rota Kops and H Herzog, "Alternative methods for attenuation correction for pet images in mr-pet scanners," IEEE Nucl Sci Conf $R$, pp. 4327-4330, Jan 2007.

[6] V Keereman, Y Fierens, T Broux, Y De Deene, M Lonneux, and $\mathrm{S}$ Vandenberghe, "Mri-based attenuation correction for pet $/ \mathrm{mri}$ using ultrashort echo time sequences," J Nucl Med, vol. 51, pp. 812-8, Jan 2010.

[7] P Mollet, V Keereman, and S Vandenberghe, "Simultaneous emission and transmission imaging for pet-mri using time-of-flight information," J Nucl Med, vol. 51 Suppl 2, pp. 124, Jan 2010.

[8] V Keereman, R Van Holen, P Mollet, and S Vandenberghe, "The effect of errors in segmented attenuation maps on pet quantification," Med Phys, vol. 38, pp. 6010-9, 2011.

[9] C Burger, G Goerres, S Schoenes, and A Buck, "Pet attenuation coefficients from ct images: experimental evaluation of the transformation of ct into pet 511-kev attenuation coefficients," Eur J Nucl Med, Jan 2002.

[10] A Loening and SS Gambhir, "Amide: a free software tool for multimodality medical image analysis," Mol Imaging, vol. 2, no. 3, pp. 131-7, Jul 2003.

[11] S Vandenberghe, Y D'Asseler, and M Koole, "Iterative list mode reconstruction for coincidence data of gamma camera," Proc SPIE, Jan 2000. 